\title{
Biogenesis and function of extracellular miRNAs
}

\author{
Chihao Zhao, Xinlei Sun and Limin Li
}

\begin{abstract}
Mature microRNAs (miRNAs) are 18-24-nucleotide non-coding RNAs with post-transcriptional regulatory functions and have been documented as an essential cornerstone of the genetic system. Although the traditional idea suggests that RNA molecules cannot be stable in extracellular environments due to ubiquitous ribonuclease, miRNA has now been verified as circulating in various body fluids in a stable, cell-free form. By associating with microvesicles (MVs) or RNA-binding proteins, extracellular miRNAs can be actively secreted and transferred into recipient cells, where they regulate target genes. Importantly, extracellular miRNAs have been demonstrated as participating in various physiological and pathological processes in bodies and have significant roles in fetal-maternal crosstalk and cross-kingdom regulation. Furthermore, the abnormal expression of extracellular miRNAs has been shown to be associated with many diseases, making extracellular miRNAs promising novel noninvasive diagnostic markers. In this review, we summarize the recent literature on the biogenesis, delivery and uptake of extracellular miRNAs, elaborate on the regulating function of extracellular miRNAs between different cells and between individuals and highlight their therapeutic potential in clinical applications.
\end{abstract}

Keywords: Extracellular miRNA, Microvesicle, Exosome, HDL, Biomarker, Crosstalk

\section{Introduction}

MicroRNAs (miRNAs) are small non-coding RNA fragments with 18-24 nucleotides in length, which can influence gene expression via post-transcriptional regulation [1-3]. Since their first discovery in Caenorbabditis elegans in the early 1990s [4], thousands of miRNAs have been reported in a variety of organisms, from single-cell algae to humans [1-3]. In recent years, increasing numbers of studies suggest that miRNAs have a high evolutionary conservation in different species, and can function as ancient and crucial cellular regulatory elements [1-3]. According to the prediction made by bioinformatics, more than half of the human proteincoding genes are expected to be controlled by miRNAs [5]. These small RNA fragments will introduce a new layer of gene regulation in eukaryotes.

The canonical miRNA biogenesis pathway in cells has been well established [1-3]. Most miRNAs are processed from primary miRNA transcripts (pri-miRNA), which

\footnotetext{
* Correspondence: liminli@nju.edu.cn

State Key Laboratory of Pharmaceutical Biotechnology, Jiangsu Engineering Research Center for MicroRNA Biology and Biotechnology, NJU Advanced Institute for Life Sciences (NAILS), School of Life Sciences, Nanjing University, 163 Xianlin Road, Nanjing 210046, Jiangsu, China
}

are $>200$ nucleotides in length and are synthesized by RNA polymerase II. Pri-miRNAs are then processed in two steps. Two members of the RNase III family of enzymes, Drosha and Dicer, catalyze the two processes. The Drosha-DGCR8 complex first cleaves pri-miRNAs into 70-nucleotide hairpin intermediates called miRNA precursors (pre-miRNA), which then are exported from the nucleus to the cytoplasm by exportin-5. In the cytoplasm, Dicer cuts the exported pre-miRNAs into 22-nucleotide miRNA/miRNA" duplexes. Finally, the guide strand of the duplex is incorporated into the RNA-induced silencing complex (RISC), while the miRNA" strand is released and degraded. As an important molecule in RNA interference, miRNAs can target the 3' untranslated regions (3' UTRs) of message RNAs (mRNAs) and induce mRNA degradation or translational inhibition, therefore suppressing protein synthesis and gene expression [1-3]. MiRNAs play critical roles in various kinds of biological processes, such as cellular development and differentiation, metabolism, proliferation, migration and apoptosis via this post-transcriptional regulation mechanism [1-3], and their altered expression is associated with many human pathologies [6-8]. 
In addition to playing a regulatory role within the cells, studies show that miRNAs can present in the plasma and serum samples in a remarkably stable form $[9,10]$. Furthermore, in other body fluids, such as saliva [11], urine [12], milk [13] and cell culture supernatants [14, 15], stable miRNAs have also been detected. These results suggest that miRNA can be stabilized in the extracellular space. More importantly, like intracellular miRNAs, these extracellular miRNAs have been found to play a regulatory role during physiological and pathological processes, and their expression profiles in body fluids are tightly related to various pathological conditions [9-12]. These findings suggest an important role of extracellular miRNAs in organisms' activities and demonstrate their potential as a diagnostic marker of disease. In this review, we summarize the relevant studies on the origin and function of extracellular miRNAs and discuss their biological significance as diagnostic markers and therapeutic drugs.

\section{The origin of extracellular miRNAs}

It has been reported that blood cells and other organs can contribute to extracellular miRNAs. Pritchard et al. analyzed 79 solid tumor circulating miRNA biomarkers reported in the literature and found that $58 \%$ of them were highly expressed in one or more blood cell type. Moreover, they showed that the plasma miRNA biomarkers' levels were tightly correlated with relevant blood cell counts or hemolysis, suggesting that serum/ plasma miRNAs are mainly derived from blood cells [16]. Specific tissue-enriched miRNAs, such as liverenriched miR-122, muscle-enriched miR-133, heartenriched miR-208 and brain-enriched miR-124, have also been detected in plasma [17-20]. Such miRNAs are released to the extracellular space via three different pathways: (1) passive leakage from broken cells caused by tissue injury, inflammation, cell necrosis or apoptosis. (2) Active secretion via membrane-enclosed cell fragments called microvesicles (MVs), which include shedding vesicles and exosomes and are released by almost all cell types under physiological and pathological conditions [21-25]. (3) Active secretion via an MV-free, RNA-binding protein-dependent pathway. Recent studies have suggested that many RNA-binding proteins, including high-density lipoprotein (HDL) [26], Argonaute 2 (AGO2) $[27,28]$ and nucleophosmin 1 (NPM1) [29], could combine with miRNAs and deliver them outside of cells. Compared with passive leakage, secreting miRNAs via MVs and HDL-binding is active and needs energy. The extracellular miRNAs secreted by active pathways are thought to play a regulatory role in biological processes.

\section{The delivery of extracellular miRNAs Delivery via MVs}

It has been shown that lipids, proteins and nucleic acids, including miRNAs, can be exchanged by MVs, giving them the ability to target recipient cells [21-25]. The membrane structure of MVs can protect miRNAs from degradation by environmental RNases and help them present stably in the extracellular space [14, 15]. Two major MVs can package and deliver miRNAs: shedding vesicles and exosomes. As a heterogeneous population, shedding vesicles are larger; 100 to $1000 \mathrm{~nm}$ in diameter vesicles are released from the plasma membrane through outward budding and fission, [21] while exosomes are $30-100 \mathrm{~nm}$ in diameter and are released by exocytosis from multivesicular bodies (MVBs) [22-25]. Evidence has accumulated that miRNAs can be packaged into MVs selectively; that is, cells may preferentially select the particular miRNA populations and sort them into MVs. For example, as the response to various stimuli, miR-150 in human blood cells and cultured THP-1 cells was selectively packaged into MVs and was actively secreted. [30] Several studies also observed higher levels of certain miRNAs in exosomes than in donor cells when the total RNA or protein content was normalized. $[14,15,31]$ Moreover, in 2013, Villarroya-Beltri et al. demonstrated that $\mathrm{T}$ cells preferred to sort several miRNAs into exosomes and that this phenomenon was maintained under resting and activated cellular conditions [32]. Further studies confirmed that a special EXOmotif GGAG present in miRNAs controlled the loading of these miRNAs into exosomes. The protein heterogeneous nuclear ribonucleoprotein A2B1 (hnRNPA2B1) could recognize the EXOmotif and specifically bind exosomal miRNAs, thus controlling their loading into exosomes [32]. Interestingly, sumoylation of hnRNPA2B1 in exosomes was essential for the binding of hnRNPA2B1 to miRNAs [32].

In recent years, the miRNA secretory mechanism has been partially revealed. By researching the release of HEK293 cells-derived exosomal miRNAs, Kosaka et al. found an actively regulated secretory machinery [33], which was ceramide-dependent and could induce endosome sorting into the exocytic MVBs [34]. A rate-limiting enzyme of ceramide biosynthesis called neutral sphingomyelinase 2 (nSMase2) acted as the key molecule in this secretion pathway and regulated the secretion of exosomal miRNAs [33]. It was supposed that nSMase 2 might increase miRNA secretion by increasing the amount of released exosomes or packaged miRNAs. However, this exosomal miRNA releasing machinery is independent of the endosomal sorting complex required for transport (ESCRT) system [33], which is important for exosomes being targeted to lysosomes [35, 36]. Taken together, these results show that a ceramide-dependent, 
ESCRT-independent pathway can regulate the incorporation of miRNAs into exosomes and their subsequent release. The mechanism of the secretion of miRNAs via shedding vesicles still unknown, and how specific miRNAs reach the vesicle shedding sites at the plasma membrane also needs further illumination.

\section{Delivery via RNA binding proteins}

Recent studies have reported that HDL, which can deliver excess cellular cholesterol to be returned to the liver for excretion, can transport endogenous miRNAs $[26,37]$. The exact process of miRNA loading onto HDL is still unclear, but previous studies showed that small RNAs could complex with zwitterionic liposomes, specifically phosphatidylcholine (PC), which are the specific lipids found on lipoproteins [38]. Vickers et al. suggested that the connection between extracellular plasma miRNAs and HDL depended on divalent cation bridging, leading to a tight association and possibly protecting miRNAs from external RNases [26]. Strikingly, the HDL-transporting process is negatively regulated by the nSMase2-mediated ceramide pathway. In other words, nSMase 2 and the ceramide pathway repress HDL-mediated miRNA exportation [26]. Given that the nSMase2-regulated ceramide pathway can promote the release of exosomal miRNAs, [33] these two pathways for the export of specific miRNAs may be opposing mechanisms, although both pathways are regulated by the same molecules.

Several pieces of evidence have shown that most extracellular miRNAs, including MV-encapsulated miRNAs, are associated with RNA-binding proteins. As important components of RISC, GW182 and AGO2, which are required for miRNA function, have been detected as having significant enrichment in exosomes [30, 39]. Moreover, our findings have demonstrated that MV-encapsulated miRNAs could enhance their resistance to RNaseA by interacting with AGO2. In addition, AGO2 could facilitate the packaging of intracellular miRNAs into MVs, and MV-loaded AGO2 is key to the function of secreted miRNAs in recipient cells [40, 41]. All these results make us realize that the loading of miRNAs into MVs is controlled by specific proteins of the RISC, and these MV-encapsulated RISC proteins, particularly AGO2, may enhance the function of extracellular miRNAs. Other RNA-binding proteins, such as NPM1, can bind extracellular miRNAs and protect them from degradation without MV-packaging [29]. Studies have demonstrated that large portions of the extracellular miRNAs are vesicle-free, protein-binding miRNAs [27, 28]. These miRNAs may be supposed to be by-products of cell death. Whether they can be delivered into recipient cells and whether they have any particular biological function remain unknown.

\section{The uptake of extracellular miRNAs}

Our previous study showed that miR-150 in THP-1 cells can be sorted into MVs and delivered into human HMEC-1 cells to regulate c-Myb expression, thus enhancing HMEC-1 cell migration [30]. This result suggests that extracellular MV-encapsulated miRNAs can be transported into specific recipient cells and play a regulatory role. The mechanism by which these miRNAs complete this process is an important issue for further research. It is thought that cells can recognize specific MVs through the recognition of surface molecules and internalize MVs by phagocytosis, endocytosis, or direct fusion with the plasma membranes, thus taking up MV-enclosed extracellular miRNAs.

The HDL-binding miRNAs can also be delivered to recipient cells. The research by Vickers et al. has suggested that the scavenger receptor $\mathrm{BI}(\mathrm{SR}-\mathrm{BI})$ is indispensable for the entrance of miRNAs into recipient cells via HDL transportation [26]. By means of SR-BI-mediated transfer, HDL-miRNAs may be delivered into the cytoplasm rather than into lysosomes, which avoids their degradation and improves their stable existence [26]. Furthermore, they have found that in recipient cells, the HDL-miRNAs have increased functional integrity and potential to alter gene expression [26]. However, a study from Wagner et al. showed that endothelial cells, which were detected as expressing the SR-BI receptor [42], internalized HDL-bound miRNAs inefficiently [37]. In addition to endothelial cells, smooth muscle cells (SMCs) and peripheral blood mononuclear cells (PBMCs) also have an inefficient uptake of HDL-miRNAs, suggesting that the regulating functions of this HDL-associated pool of miRNAs in these cells may not be of major importance [37]. Collectively, these results indicate that the questions of whether HDL-miRNA transfer is ubiquitous in various cell types need more investigation.

\section{The function of extracellular miRNAs}

The widespread presence of extracellular miRNAs and their stability in circulation suggest a fascinating function of these cell-derived miRNAs. However, we still know little about the function of most secreted miRNAs, particularly MV-free, protein-binding extracellular miRNAs. The role of MV-encapsulated miRNAs in regulating physiological and pathological processes has been explored and partially uncovered in recent years. That miRNAs can be transferred via MVs and can subsequently play functional roles in recipient cells have been proven by several groups. These miRNAs appear to be a novel component for intercellular communication, and the cross-talk mediated by extracellular miRNAs may provide a new angle to understand the mechanisms of dysfunctional conditions. 


\section{Extracellular miRNAs are involved in tumor progression}

Many tumors can proactively manipulate their surrounding microenvironment, thus promoting their survival, growth and invasion. For instance, cancer cells can convert the surrounding normal cells from their original homeostatic states into the neoplastic nature of the tumor through secreted molecules and paracrine signaling. The communication between cancer cells and normal cells in microenvironments have been proven crucial to cancer progression [43]. Recent studies have discovered that extracellular miRNAs from cancer cells can target their surroundings and are deeply involved in tumorigenesis. Umezu et al. showed that exosomal miR-92a, derived from the leukemia cells K562, transferred into human umbilical vein endothelial cells (HUVECs), directly regulated the target gene integrin $\alpha 5$, leading to enhanced endothelial cell migration and tube formation [44]. Our research in 2014 also demonstrated that tumor-secreted miR-214 was sufficiently delivered into targeted mouse peripheral $\mathrm{CD}_{4}^{+} \mathrm{T}$ cells by MVs and efficiently downregulated phosphatase and tensin homolog (PTEN), causing the promotion of Treg expansion [45]. The cancer cell-secreted miR-214-mediated Treg expansion could further suppress the immune system and enhance tumor implantation/growth in mice. This phenomenon could be reversed after MV delivery of anti-miR-214 antisense oligonucleotides (ASOs) to tumors [45]. These results sufficiently suggest that cancer cells actively package miRNAs into MVs and transfer them to their surroundings to change tumor-stromal environments, thus promoting their survival and malignancy. Recently, the mechanisms governing tumor exosome release were partially uncovered by our group. We found that pyruvate kinase type M2 (PKM2), an enzyme associated with tumor cell reliance on aerobic glycolysis, could promote tumor cell exosome release [46]. In detail, PKM2 was upregulated and phosphorylated in tumors, and the phosphorylated PKM2 served as a protein kinase to phosphorylate synaptosome-associated protein 23 (SNAP-23) at Ser95. This in turn enabled the formation of the soluble N-ethylmaleimide-sensitive fusion factor attachment protein receptor (SNARE) complex to allow the release of exosomes [46]. Collectively, it has been widely suggested that as the regulators secreted actively by tumor cells, tumor-derived extracellular miRNAs have a significant impact on the surrounding normal organizations, thus promoting the development of cancer.

\section{Extracellular miRNAs as potential diagnostic biomarkers}

In the field of cancer and other disease diagnosis, extracellular miRNAs have also served as novel biomarkers. Lawrie et al. first described that miR-155, miR-210 and miR-21 had the potential to be non-invasive diagnostic markers for diffuse large B-cell lymphoma (DLBCL). In addition, they observed that miR-21 expression in serum was associated with relapse-free survival in DLBCL patients [47]. A series of follow-up studies supported Lawrie's opinion and found that extracellular miRNAs could be clinical biomarkers for the diagnosis and prognosis of many diseases, including cancers [9-12], diabetes [10] and viral infections [48, 49]. Compared to other traditional biomarkers, extracellular miRNAs have four major advantages: (1) Extracellular miRNAs can be stable in RNase-enriched human body fluids. (2) The sampling process is non-invasive and does not require tissue samples. (3) Many detection methods, such as qRT-PCR, deep sequencing and microarray, can easily detect extracellular miRNAs in a quantitative manner. (4) The changes in extracellular miRNA profiles are sensitive to the development of the disease, especially at an early stage. However, the precise analysis of extracellular miRNAs still requires consideration of many critical influenced preanalytical and analytical variables. Sample collection, RNA extraction and detection processes also need further exploration and normalization. Taken together, the road to transform extracellular miRNAs research achievements to clinical applications is still long.

\section{Extracellular miRNAs as therapeutic drugs}

MiRNAs and siRNAs can target specific genes and regulate protein expression levels. Thus, by modulating abnormally expressed disease genes, these RNA molecules seem to show excellent potential as therapeutics for various diseases $[50,51]$. The key to the successful application of small RNAs in clinical practice is their efficient delivery $[50,51]$. Traditional small RNA delivery vehicles using viruses and liposomes have high toxicity and low efficiency due to rejection reactions. In contrast, as membranous vesicles secreted by almost all cell types, MVs can naturally fuse with plasma membranes and transfer packaged functional small RNAs into cells in a way that is better tolerated by the immune system. Therefore, MVs may become a potentially delivery vehicle with low toxicity and high efficiency for drug RNAs. There is some evidence showing that MVs can deliver drug siRNAs to specific target cells for therapeutic effects. Alvarez-Erviti et al. prepared exosomes from dendritic cells (DCs), which expressed the fusion of an exosomal membrane protein Lamp2b and the neuron-specific rabies viral glycoprotein (RVG) peptide and intravenously injected these siRNA-loaded exosomes into mice [52]. They found that the packaged siRNA in these RVG-targeted exosomes could be directionally delivered to neurons, oligodendrocytes and microglia in mouse brains, leading to a specific gene knockdown [52]. Moreover, they loaded the siRNA of 
BACE1, a therapeutic target in Alzheimer's disease into the RVG-targeted exosomes and delivered them into wild-type mice via intravenous injection. The results showed a significant mRNA and protein downregulation of BACE1 in mice brains, suggesting the therapeutic potential of this exosome-mediated siRNA delivery system [52]. In addition, our group prepared another exosome loaded with opioid receptor mu (MOR) siRNA using similar exosomes with RVG and found that these exosomes could efficiently deliver the siRNA to the mouse brain, significantly reducing MOR mRNA and protein levels and strongly inhibiting morphine relapse in mice [53]. Interestingly, we demonstrated that the drug MOR siRNAs were also associated with AGO2 in RVG exosomes [53]. Taken together, these results note that exosomes can deliver small RNAs to specific targets, and this character of exosome-transported RNAs will open a new avenue for therapeutic applications.

\section{Extracellular miRNAs as a new tool for crosstalk between mothers and their children}

It has been suggested that extracellular miRNAs also play their regulatory roles between mother and fetus. Through the research on pregnant mice, we mentioned that the exogenous miRNAs assimilated by mothers could be absorbed by the fetus via transplacental transmission, thereby influencing its growth and development. We gavage-fed synthetic exogenous influenza virus miRNAs and honeysuckle (HS) decoction enriched with exogenous plant MIR2911 to pregnant mice that had mature placentas (usually pregnant for at least 14 days) and found the significant elevation of these exogenous miRNAs not only in the maternal plasma, but, more importantly, in the fetal liver [54]. Measuring the amount of circulating MIR2911 in MVs compared to that in MV-free plasma, we then suggested that these circulating miRNAs were primarily MV-loaded and that an MV-mediated pathway may affect their transmission through the placenta [54]. Meanwhile, we similarly fed the siRNA of alpha-fetoprotein (AFP) to pregnant mice and found the siRNAs in the fetal liver. Moreover, the mRNA and protein levels of AFP were dramatically down-regulated in the fetal mice. The analogical phenomenon was observed when MV-packaged siRNAs were injected directly into maternal mice [54]. In this study, we hypothesized that fetal development during pregnancy could be influenced by endogenous/exogenous small non-coding RNAs, including miRNAs and siRNAs, via their transference through the placenta [54]. In summary, the investigation of extracellular miRNAs in pregnancies may suggest another important index assessing fetal development and health status and a potentially effective tool for treating fetal diseases before birth.
The effect of extracellular miRNAs on the communication between mothers and their children can also be done by breastfeeding. Kosaka et al. showed the existence of miRNAs in human milk by performing a miRNA microarray and analyzed their expression profiles. They found that several immune-related miRNAs, such as miR-155 (regulates the innate immune response and Tand B-cell maturation), miR-125b (suppresses TNF- $\alpha$ production), miR-223 (important for neutrophil proliferation and activation) and let-7i (influences the expression of Toll-like receptor 4 in human cholangiocytes), were abundant in the milk [13]. Notably, these immune system-related miRNAs had higher expression in the first 6 months after delivery, which is the period during which infants receive only breast milk [13]. Similar results were demonstrated by our research focused on bovine colostrum, and we further discovered that extracellular miRNAs may be stabilized in milk by associating with MVs [55]. In the follow-up study, Liao et al. used a simulated gastric/pancreatic digestion protocol to perform an in vitro digestion of human milk exosomes and found that the overall miRNA abundance in human milk exosomes was stable after digestion [56]. Surprisingly, by nuclear localization, the authors showed that the in vitro digested human milk exosomes could be internalized by human intestinal epithelial crypt-like cells (HIEC) [56]. Summarizing all these results, we propose the hypothesis that extracellular functional miRNAs in breast milk exosomes may be transferred from the mother to the infants through lactation and may be absorbed by infants after digestion, thus affecting the immune response and development of the infants.

\section{Extracellular miRNAs as novel nutriments or virulence factors within the interaction of species}

Many species of plants are the main source of nutrition for animals. Through food intake and digestion, the various nutrients of the plants, including carbohydrates, proteins, minerals and vitamins can be absorbed and transferred into the circulatory systems of the animals. Large amounts of evidence have demonstrated that miRNAs are abundant in plants and are indispensable in regulating plants' physiological activities. As an important part of the plants, whether these functional plants miRNAs can exist and function in animals after digestion needs to be explored. In 2012, we reported that exogenous plant miRNAs could be detected in various animals' plasma and tissues, where they could regulate the expression of mammalian target genes [57]. In detail, we analyzed the global miRNA expression profile in the serum of healthy Chinese donors and found $\sim 30$ known plant miRNAs. Among them, MIR156a and MIR168a had substantial expression levels. These two plant miRNAs were also detected in serum samples from several 


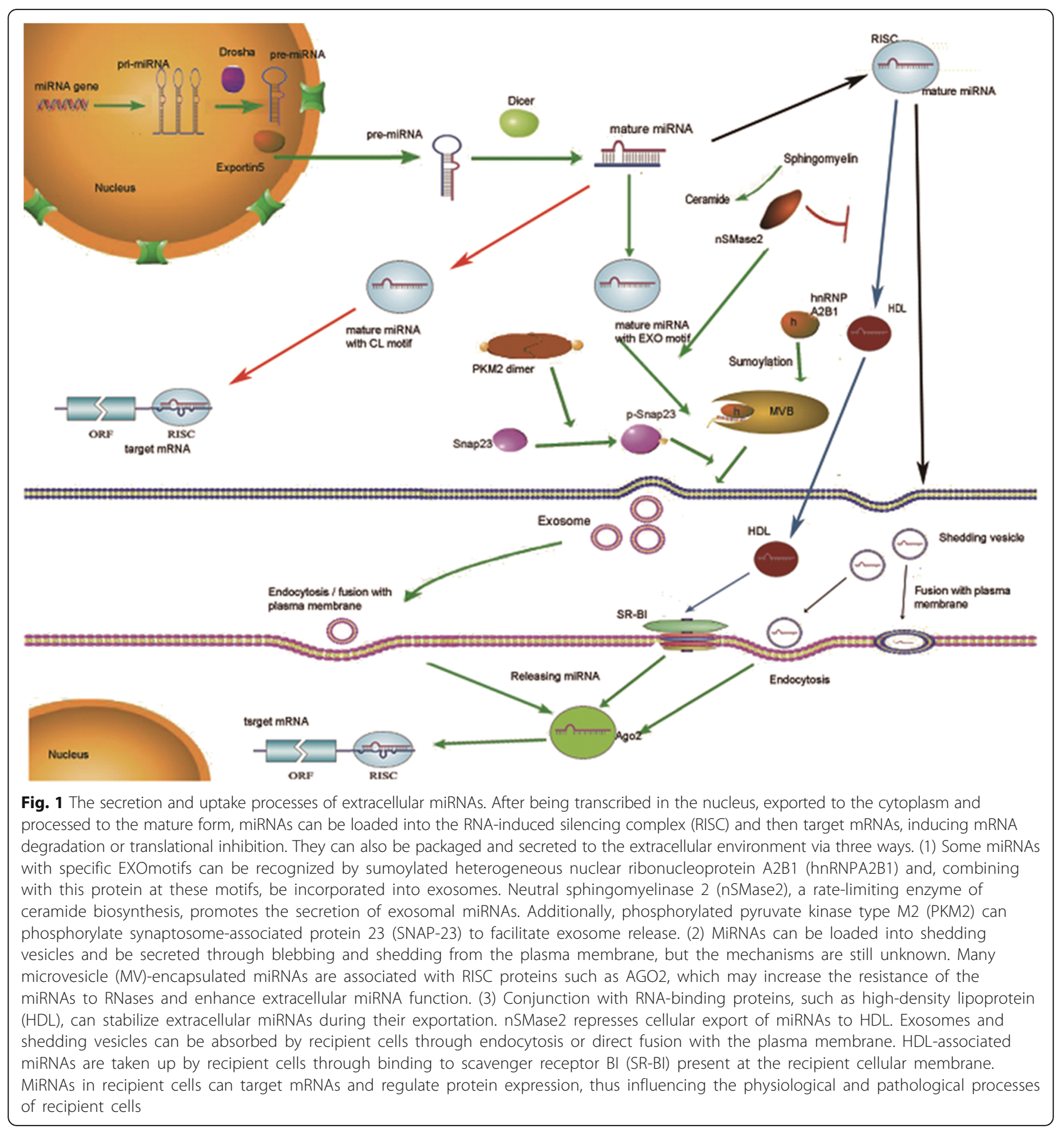

kinds of animals and in organs from mice [57]. Furthermore, we found that MIR156a and MIR168a could resist digestion and traverse the mouse gastrointestinal (GI) tract, eventually entering the mouse's circulatory system and reaching various organs. The 2'-O-methylation of the plant miRNAs and the association with MVs may protect them from degradation during digestion and enhance their stability in circulation [57]. Further functional studies showed that exogenous plant MIR168a could combine with the human/mouse low-density lipoprotein receptor adapter protein 1 (LDLRAP1) mRNA and repress the expression of LDLRAP1 in the liver, thus decreasing the removal of low-density lipoprotein (LDL) from mouse plasma [57]. Given that the liver-enriched LDLRAP1 protein could facilitate removal of LDL from circulation and that MIR168a was enriched in rice, which is the main source of food for Chinese people, we suggested that the rice-based daily diet may influence liver lipid metabolism through the cross-kingdom regulation between MIR168a and the 
LDLRAP1 in Chinese people. Similar results have been detected by a subsequent study focused on Western donors from Chin et al. [58]. All of these findings showed that exogenous miRNAs can be assimilated by the GI tract and can be delivered to various organs through food intake, digestion and circulation. Like endogenous miRNAs, these exogenous miRNAs also have bioactivities and regulate the target genes. The exogenous miRNAs from dietary sources may become a novel nutrient component, such as minerals and vitamins.

Recent studies have showed that specific extracellular miRNAs act as the virulence factors in host-pathogen interactions during infection. For example, some viruses can hijack host cells' MV generation and secretion pathways to spread their own miRNAs, thus silencing the expression of immunoregulatory genes in the recipient cells. Pegtel et al. reported that the first known miRNA-encoding virus, the Epstein-Barr virus (EBV) [59], can secrete viral miRNAs from infected B cells through exosomes [60]. These exosomal viral miRNAs were then delivered into monocyte-derived dendritic cells $(\mathrm{MoDC})$ and repressed the immunoregulatory gene CXCL11/ITAC in a dose-dependent manner. The downregulation of CXCL11/ITAC has been detected in primary EBV-associated lymphomas [60]. The analogical phenomenon existed in the plant kingdom. A research from Shahid et al. showed that the parasitic plant Cuscuta campestris could accumulate high levels of miRNAs while parasitizing host plants and deliver them to host through its haustoria [61]. In host plants, these parasite-derived miRNAs hijacked the host's own silencing machinery to produce secondary siRNAs, thereby remodeling host gene expression to the parasite's advantage [61]. To sum up all these findings, we suggest that extracellular miRNAs play indispensable roles in the host-pathogen interaction during the infection. The discovery of these miRNAs reveals a new relationship between hosts and parasites, providing a new approach for the treatment of infectious diseases.

\section{Conclusions}

In this review, we summarize that extracellular miRNAs are present in various body fluids stably through MV encapsulation or by binding with proteins, and they can be actively secreted and transported and finally can bind to and regulate target genes in recipient cells (Fig. 1). By influencing physiological and pathological processes of target cells, extracellular miRNAs have been widely demonstrated as regulators involved in cell-cell communication, fetal-maternal crosstalk and even cross-kingdom regulation. In addition, as diagnostic biomarkers and treatments, the potential clinical applications of extracellular miRNAs have been investigated.
The current discoveries regarding extracellular miRNAs are undoubtedly exciting. However, many unanswered questions still exist in this research field. For example, although a motif controlling intracellular miRNAs loaded into exosomes has been detected [32], the intracellular sorting mechanisms of secreted miRNAs still have many unknown factors. Whether there are other motifs associated with exosome packaging of miRNAs and how specific miRNAs are sorted and packaged into shedding vesicles or bound with HDL require extensive exploration. Another question needing to be answered is how donor cells regulate their secreted miRNAs to target specific genes, as multiple transcripts can be combined by one miRNA in recipient cells. Moreover, differences in measurement methodologies and random degradation make the accurate quantification of exosome-derived miRNAs difficult. Several barriers to the delivery of circulating miRNA antagomirs or mimics, such as low payload capability, limited tissue permeability and poor bioavailability, may also influence the therapeutic effects of extracellular miRNAs. Thus, it is essential to explore more accurate quantitative methods and more efficient delivery mechanisms for extracellular miRNAs in further studies. Nevertheless, this emerging field may extend our understanding of the communication between cells and between individuals. The elucidation of extracellular miRNAs will be of great significance in helping us better understanding the biological processes of organisms.

\section{Abbreviations \\ 3'UTR: 3' untranslated region; AGO2: Argonaute 2; HDL: High-density lipoprotein; miRNA: microRNA; mRNA: Message RNA; MV: Microvesicle; NPM1: Nucleophosmin 1; nSMase2: Neutral sphingomyelinase 2; RISC: RNA- induced silencing complex; SR-BI: Scavenger receptor BI}

\section{Acknowledgements}

Not applicable.

Funding

This work was supported by grants from Natural Science Foundation of Jiangsu Province (BK20170076), Six talent peaks project of Jiangsu Province, National Natural Science Foundation of China (31670917) and the Fundamental Research Funds for the Central Universities (020814380039 and 020814380082).

\section{Availability of data and materials} Not applicable.

\section{Authors' contributions}

CZ made a major contribution in the manuscript writing process. $\mathrm{LL}$ contributed to writing and revising the manuscript. XS drew the figure. All authors read and approved the final manuscript.

Ethics approval and consent to participate Not applicable.

Consent for publication

Not applicable.

Competing interests

The authors declare that they have no competing interests. 


\section{Publisher's Note}

Springer Nature remains neutral with regard to jurisdictional claims in published maps and institutional affiliations.

Received: 7 December 2017 Accepted: 8 April 2019

Published online: 26 April 2019

\section{References}

1. Pasquinelli AE. MicroRNAs and their targets: recognition, regulation and an emerging reciprocal relationship. Nat Rev Genet. 2012;13(4):271-82.

2. Pelaez N, Carthew RW. Biological robustness and the role of microRNAs: a network perspective. Curr Top Dev Biol. 2012;99:237-55.

3. Kim VN, Han J, Siomi MC. Biogenesis of small RNAs in animals. Nat Rev Mol Cell Biol. 2009;10(2):126-39.

4. Lee RC, Feinbaum RL, Ambros V. The C. elegans heterochronic gene lin-4 encodes small RNAs with antisense complementarity to lin-14. Cell. 1993; 75(5):843-54.

5. Friedman RC, et al. Most mammalian mRNAs are conserved targets of microRNAs. Genome Res. 2009;19(1):92-105

6. Esquela-Kerscher A, Slack FJ. Oncomirs - microRNAs with a role in cancer. Nat Rev Cancer. 2006;6(4):259-69.

7. Calin GA, Croce CM. MicroRNA signatures in human cancers. Nat Rev Cancer. 2006;6(11):857-66.

8. Cho WCS. MicroRNAs: potential biomarkers for cancer diagnosis, prognosis and targets for therapy. Int J Biochem Cell Biol. 2010;42(8):1273-81.

9. Mitchell PS, et al. Circulating microRNAs as stable blood-based markers for cancer detection. Proc Natl Acad Sci U S A. 2008;105(30):10513-8.

10. Chen $X$, et al. Characterization of microRNAs in serum: a novel class of biomarkers for diagnosis of cancer and other diseases. Cell Res. 2008;18(10): 997-1006.

11. Park NJ, et al. Salivary microRNA: discovery, characterization, and clinical utility for oral cancer detection. Clin Cancer Res. 2009;15(17):5473-7.

12. Hanke $M$, et al. A robust methodology to study urine microRNA as tumor marker: microRNA-126 and microRNA-182 are related to urinary bladder cancer. Urologic Oncol Semin Orig Investig. 2010;28(6):655-61.

13. Kosaka $\mathrm{N}$, et al. microRNA as a new immune-regulatory agent in breast milk Silence. 2010;1(1):7.

14. Skog J, et al. Glioblastoma microvesicles transport RNA and proteins that promote tumour growth and provide diagnostic biomarkers. Nat Cell Biol. 2008;10(12):1470-U209.

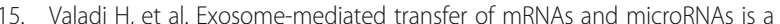
novel mechanism of genetic exchange between cells. Nat Cell Biol. 2007; 9(6):654-U72.

16. Pritchard CC, et al. Blood cell origin of circulating MicroRNAs: a cautionary note for cancer biomarker studies. Cancer Prev Res. 2012;5(3):492-7.

17. Laterza OF, et al. Plasma MicroRNAs as sensitive and specific biomarkers of tissue injury. Clin Chem. 2009:55(11):1977-83.

18. Corsten MF, et al. Circulating MicroRNA-208b and MicroRNA-499 reflect myocardial damage in cardiovascular disease. Circ Cardiovasc Genet. 2010; 3(6):499-506.

19. Lewis AP, Jopling CL. Regulation and biological function of the liver-specific miR-122. Biochem Soc Trans. 2010;38:1553-7.

20. Zhang $Y$, et al. Plasma MicroRNA-122 as a biomarker for viral-, alcohol-, and chemical-related hepatic diseases. Clin Chem. 2010;56(12):1830-8.

21. Cocucci E, Racchetti G, Meldolesi J. Shedding microvesicles: artefacts no more. Trends Cell Biol. 2009;19(2):43-51.

22. Thery C, Zitvogel L, Amigorena S. Exosomes: composition, biogenesis and function. Nat Rev Immunol. 2002;2(8):569-79.

23. Mathivanan S, Ji H, Simpson RJ. Exosomes: Extracellular organelles important in intercellular communication. J Proteome. 2010;73(10):1907-20.

24. Ratajczak J, et al. Membrane-derived microvesicles: important and underappreciated mediators of cell-to-cell communication. Leukemia. 2006; 20(9):1487-95.

25. Simons M, Raposo G. Exosomes - vesicular carriers for intercellular communication. Curr Opin Cell Biol. 2009;21(4):575-81.

26. Vickers $\mathrm{KC}$, et al. MicroRNAs are transported in plasma and delivered to recipient cells by high-density lipoproteins. Nat Cell Biol. 2011;13(4): 423-U182.

27. Turchinovich $A$, et al. Characterization of extracellular circulating microRNA Nucleic Acids Res. 2011;39(16):7223-33.
28. Arroyo JD, et al. Argonaute2 complexes carry a population of circulating microRNAs independent of vesicles in human plasma. Proc Natl Acad Sci U S A. 2011;108(12):5003-8

29. Wang $K$, et al. Export of microRNAs and microRNA-protective protein by mammalian cells. Nucleic Acids Res. 2010;38(20):7248-59.

30. Zhang YJ, et al. Secreted Monocytic miR-150 enhances targeted endothelial cell migration. Mol Cell. 2010;39(1):133-44.

31. Mittelbrunn $\mathrm{M}$, et al. Unidirectional transfer of microRNA-loaded exosomes from T cells to antigen-presenting cells. Nat Commun. 2011;2:282.

32. Villarroya-Beltri $C$, et al. Sumoylated hnRNPA2B1 controls the sorting of miRNAs into exosomes through binding to specific motifs. Nat Commun. 2013:4:2980

33. Kosaka N, et al. Secretory mechanisms and intercellular transfer of MicroRNAs in living cells. J Biol Chem. 2010;285(23):17442-52

34. Trajkovic $K$, et al. Ceramide triggers budding of exosome vesicles into multivesicular endosomes. Science. 2008;319(5867):1244-7.

35. Hurley JH. ESCRT complexes and the biogenesis of multivesicular bodies. Curr Opin Cell Biol. 2008;20(1):4-11

36. Williams RL, Urbe $S$. The emerging shape of the ESCRT machinery. Nat Rev Mol Cell Biol. 2007;8(5):355-68.

37. Wagner J, et al. Characterization of levels and cellular transfer of circulating lipoprotein-bound MicroRNAs. Arteriosclerosis Thromb Vasc Biol. 2013;33(6): 1392-+

38. Lu D, Rhodes DG. Binding of phosphorothioate oligonucleotides to zwitterionic liposomes. Biochim Biophys Acta. 2002;1563(1-2):45-52.

39. Gibbings DJ, et al. Multivesicular bodies associate with components of miRNA effector complexes and modulate miRNA activity. Nat Cell Biol. 2009; 11(9):1143-U223.

40. Li LM, et al. Argonaute 2 complexes selectively protect the circulating MicroRNAs in cell-secreted microvesicles. PLoS One. 2012;7(10):e46957.

41. Lv ZY, et al. Argonaute 2 in cell-secreted microvesicles guides the function of secreted miRNAs in recipient cells. PLoS One. 2014;9(7):e103599.

42. Yeh YC, et al. Identification and expression of scavenger receptor SR-BI in endothelial cells and smooth muscle cells of rat aorta in vitro and in vivo. Atherosclerosis. 2002;161(1):95-103.

43. Muralidharan-Chari $\mathrm{V}$, et al. Microvesicles: mediators of extracellular communication during cancer progression. J Cell Sci. 2010;123(10):1603-11.

44. Umezu T, et al. Leukemia cell to endothelial cell communication via exosomal miRNAs. Oncogene. 2013;32(22):2747-55.

45. Yin $Y$, et al. Tumor-secreted miR-214 induces regulatory T cells: a major link between immune evasion and tumor growth. Cell Res. 2014;24(10): 1164-80.

46. Wei $Y$, et al. Pyruvate kinase type M2 promotes tumour cell exosome release via phosphorylating synaptosome-associated protein 23. Nat Commun. 2017:8:14041.

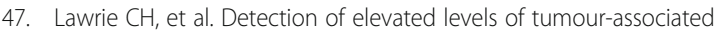
microRNAs in serum of patients with diffuse large B-cell lymphoma. $\mathrm{Br}$ J Haematol. 2008;141(5):672-5

48. Li LM, et al. Serum microRNA profiles serve as novel biomarkers for HBV infection and diagnosis of HBV-positive Hepatocarcinoma. Cancer Res. 2010; 70(23):9798-807.

49. Chen $Y Y$, et al. A pilot study of serum microRNA signatures as a novel biomarker for occult hepatitis B virus infection. Med Microbiol Immunol. 2012;201(3):389-95.

50. Ryther RC, et al. siRNA therapeutics: big potential from small RNAs. Gene Ther. 2005;12(1):5-11.

51. Weiler J, Hunziker J, Hall J. Anti-miRNA oligonucleotides (AMOs): ammunition to target miRNAs implicated in human disease? Gene Ther. 2006;13(6):496-502

52. Alvarez-Erviti $\mathrm{L}$, et al. Delivery of siRNA to the mouse brain by systemic injection of targeted exosomes. Nat Biotechnol. 2011;29(4):341-U179.

53. Liu YC, et al. Targeted exosome-mediated delivery of opioid receptor mu siRNA for the treatment of morphine relapse. Sci Rep. 2015;5:17543.

54. Li J, et al. Small non-coding RNAs transfer through mammalian placenta and directly regulate fetal gene expression. Protein Cell. 2015;6(6):391-6.

55. Sun $Q$, et al. Immune modulatory function of abundant immune-related microRNAs in microvesicles from bovine colostrum. Protein Cell. 2013;4(3): 197-210.

56. Liao $Y$, et al. Human milk exosomes and their microRNAs survive digestion in vitro and are taken up by human intestinal cells. Mol Nutr Food Res. 2017:61(11):1700082 
57. Zhang $L$, et al. Exogenous plant MIR168a specifically targets mammalian LDLRAP1: evidence of cross-kingdom regulation by microRNA. Cell Res. 2012;22(1):107-26.

58. Chin AR, et al. Cross-kingdom inhibition of breast cancer growth by plant miR159. Cell Res. 2016;26(2):217-28.

59. Pfeffer $\mathrm{S}$, et al. Identification of virus-encoded microRNAs. Science. 2004; 304(5671):734-6

60. Pegtel DM, et al. Functional delivery of viral miRNAs via exosomes. Proc Natl Acad Sci U S A. 2010;107(14):6328-33.

61. Shahid S, et al. MicroRNAs from the parasitic plant Cuscuta campestris target host messenger RNAs. Nature. 2018;553(7686):82-5.

Ready to submit your research? Choose BMC and benefit from:

- fast, convenient online submission

- thorough peer review by experienced researchers in your field

- rapid publication on acceptance

- support for research data, including large and complex data types

- gold Open Access which fosters wider collaboration and increased citations

- maximum visibility for your research: over $100 \mathrm{M}$ website views per year

At $\mathrm{BMC}$, research is always in progress.

Learn more biomedcentral.com/submissions 